RECyT

Año 22 / № 33 / 2020 / 116-123

\title{
Características oclusales estáticas y dinámicas de los estudiantes de la cátedra de oclusión dental
}

\section{Static and Dynamic Occlusal features of students of the dental occlusion Chair}

\author{
Lourdes V. Rojas Paredes ${ }^{1, *}$, Claudia Elena González ${ }^{1}$ \\ 1- Facultad de Ciencias de la Salud. Carrera de Odontología, Universidad Católica Nuestra Señora de la Asunción; Campus Itapuá, \\ Encarnación, Paraguay. \\ *E-mail: lourdes.rojas@uc.edu.py
}

Recibido el 27 de marzo de 2019, Aprobado el 16 de diciembre de 2019.

\section{Resumen}

Con este estudio se pretendió determinar las características oclusales estáticas y dinámicas de 41 estudiantes sanos, de 18 a 21 años de edad, del segundo año de la Carrera de Odontología de la Universidad Católica "Nuestra Señora de la Asunción" de Itapúa-Paraguay en los años 2017 y 2018. Se realizaron modelos completos superior e inferior montados en un articulador semiajustable. La forma de arco más frecuente para ambas arcadas fue la ovoide. Los resultados de las mediciones de Overbite y Overjet tuvieron una tendencia mayoritariamente hacia los parámetros normales. La discrepancia de la línea media está presente en la mitad de los alumnos. Los casos de mordida cruzada son infrecuentes. La clase I de angle molar y canina bilateral es la más frecuente. La desoclusión canina fue el patrón más común de movimiento lateral funcional existiendo en un bajo porcentaje interferencias del lado de no trabajo.

Palabras clave: Oclusión dental; Maloclusiones; Características oclusales.

\section{Abstract}

This study aimed at determining the static and dynamic occlusal features among 41 healthy students, who were aged 18 and 21 respectively. They were undergoing the second year of the dentistry career at the Catholic University "Nuestra Señora de la Asunción" in Itapúa-Paraguay during 2017 and 2018. Complete upper and lower models were taken and mounted on a semi adjustable articulator. The most frequent arch shape for both arcades was ovoid. The results of the measurements of both Overbite and Overjet mainly had a tendency towards the normal parameters. The midline discrepancy is present in half of the students. Cross-bite cases are rare. The molar and canine class I is the most frequent. Canine Desocclusion was the most common pattern of functional lateral movement although low percentage interferences existed on the non-working side.

Keywords: Dental occlusion; Malocclusion; Occlusal features.

\section{Introducción}

Millones de personas en todo el mundo están sufriendo de problemas orodentales a pesar de que la mayoría de ellos son prevenibles. La maloclusión es uno de ellos y no es una enfermedad, sino una variación morfológica que puede o no ser asociada a condiciones patológicas [1]. La maloclusión es un problema de salud pública que afecta a un rango que va desde el 39 al 93\% de la población mundial. Su etiología es multifactorial, considerándose la herencia y el factor ambiental los más relevantes, podríamos precisar como modificaciones en el crecimiento y desarrollo de los maxilares, así como por alteraciones a nivel dental, que generan cambios en la forma, función y estética del sistema estomatognático [2,3].

Según la Organización Mundial de la Salud-OMS las patologías de orden oclusal son las más prevalentes luego de las caries y la enfermedad periodontal dentro de las afecciones bucodentales. Igualmente, según datos de la Organización Panamericana de la Salud-OPS, el $80 \%$ de la población presenta alteraciones oclusales en Latinoamérica [4].

La mayoría de las investigaciones han defendido que el reciente aumento en la aparición de la maloclusión debe atribuirse a la disponibilidad de una dieta más procesada y a la reducción de la necesidad de una acción masticatoria poderosa u otros factores ambientales [5].

La etiología de los trastornos temporomandibulares (TTM) siempre ha sido un tema de debate, según lo sugerido por las teorías evolutivas sobre Fisiopatología de los TTM se debe abandonar conceptos enfocados en la oclusión únicamente para abrazar conceptos centrados en los pacientes con abordaje biopsicosocial. A pesar de que 
el papel de la oclusión dental como factor de riesgo para los TTM ha disminuido progresivamente a lo largo de los años, la posibilidad de que las características oclusales de un individuo puedan condicionar los efectos del bruxismo actuando como un campo de batalla a través del cual las cargas de bruxismo son ejercida sobre las estructuras estomatognáticas, por lo que representa un factor potencial que influye en el patrón de los síntomas de TTM en los bruxómanos, y no se puede subestimar [6, 7].

Por lo tanto, el papel potencial de la maloclusión en pacientes con TTM es todavía digno de exploración. Esto es especialmente cierto si se tiene en cuenta la alta prevalencia de rasgos de la supuesta maloclusión y su relevancia clínica, la cual debe ser evaluada mediante su posible asociación con alteraciones funcionales. Por eso, el enfoque se debe poner en la relación entre las diversas características de la maloclusión y las anomalías posicionales de los discos de la Articulación Temporomandibular (ATM), que son signos tempranos de la alteración biomecánica de la ATM [8]. Partiendo de esta premisa, la presente investigación se realizó con el objetivo de determinar algunas características estáticas y dinámicas de la oclusión dental en 41 alumnos del segundo año de la Universidad Católica "Nuestra Señora de la Asunción" Campus Itapúa en los años 2017-2018.

\section{Materiales y Métodos}

\section{Materiales}

Modelos de arcos superior e inferior montados en articuladores semiajustables todos de tipo arcón. Las marcas comerciales utilizadas por los alumnos fueron Dentflex
10600, Bio art 400-S y Bio art A7 Plus E. Las fichas clínicas de pacientes fueron obtenidas del libro Manual Práctico de Oclusión Dentaria [9]. Planillas Microsoft Excel. La información que fue recogida en las fichas se llevó a hojas de Microsoft Excel 2016 y los datos se ordenaron en tablas que facilitaron su procesamiento estadístico en dicho programa.

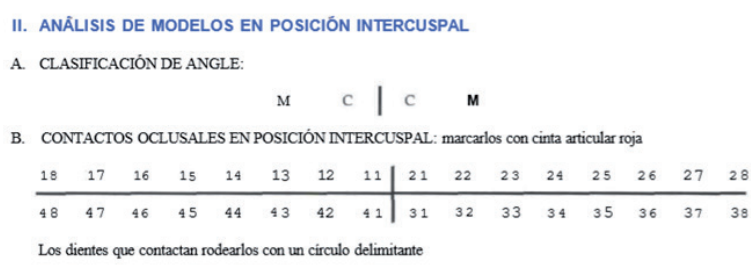

E. LINEAS MEDIAS DENTARIA Y MAXILAR Coincidencia

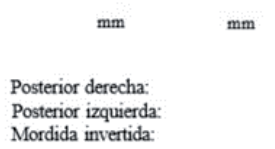

Figura 2: Ficha dental, apartado con datos analizados con los modelos en posición intercuspal.

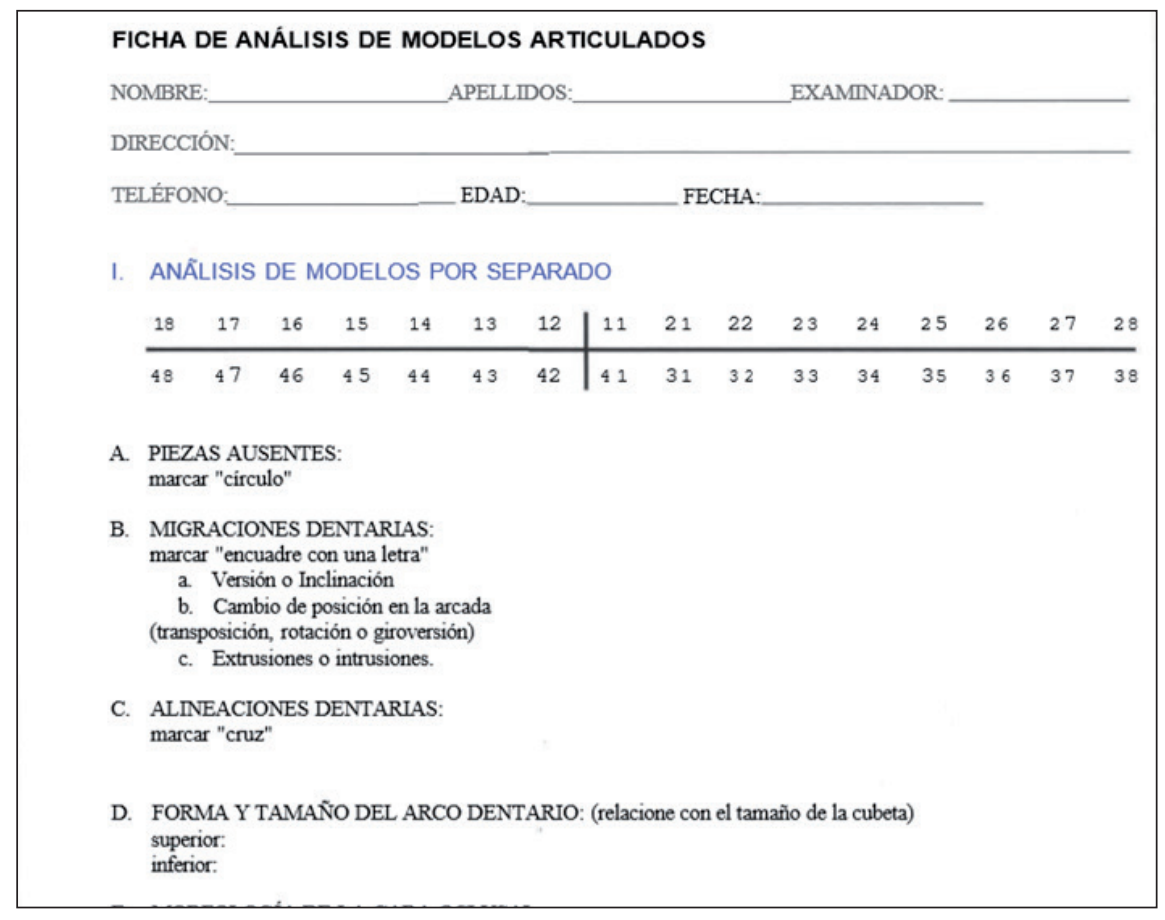

Figura 1: Ficha dental, unidad con datos observados con los modelos por separados. 


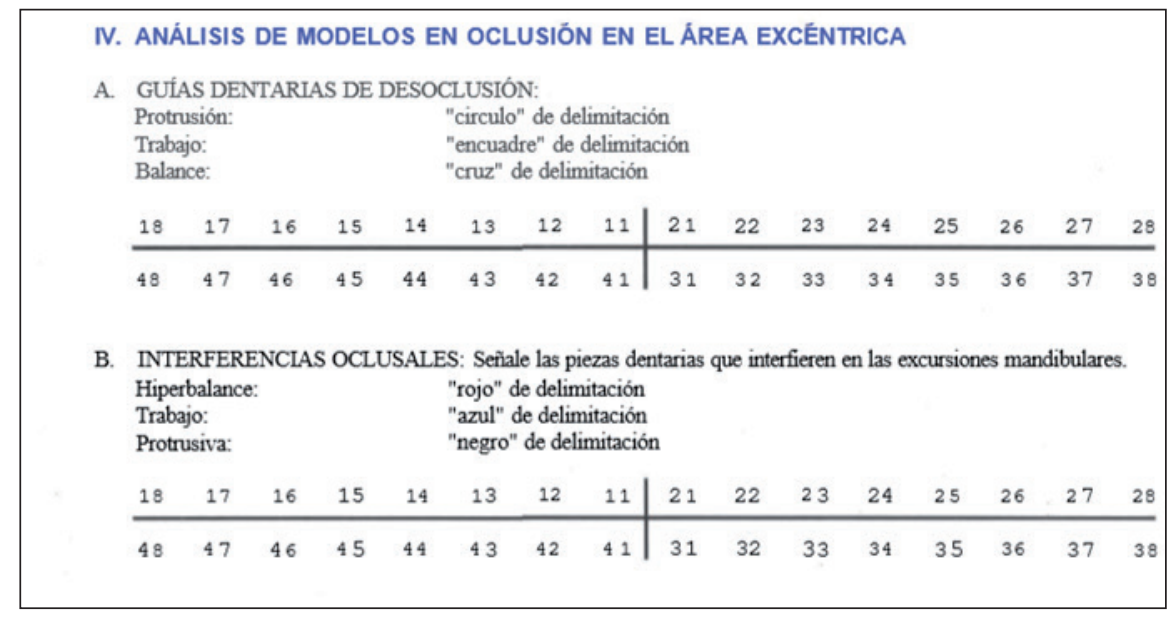

Figura 3: Ficha dental, sección con datos analizados con los modelos en movimientos excéntricos.

\section{Métodos}

Se realizaron 41 fichas clínicas de los modelos tomados a los alumnos de ambos sexos, de 18 a 21 años de edad de segundo año de la Carrera de Odontología de la Universidad Católica, Campus Itapúa, de la ciudad de Encarnación entre los años 2017 al 2018. La toma de impresión fue llevada a cabo por alumnos del segundo año, previa instrucción de la técnica apropiada en la cátedra de Oclusión de la Facultad de Ciencias de la Salud de la Carrera de Odontología. El vaciado de los modelos de trabajo se realizó con yeso extraduro tipo 4 en ambas arcadas. Para el montaje del modelo superior fue utilizado arco facial, se acondicionó compuesto de modelar y se colocó en el plano de orientación de la horquilla del arco facial, se introdujo la horquilla en la cavidad bucal del estudiante y se presionó sobre los dientes superiores de este, las olivas fueron introducidas en los conductos auditivos y se ajustaron las ramas laterales del arco facial, luego fue ajustado el nasion en la posición correcta como tercer punto de referencia anatómico. Para el montaje del modelo inferior se utilizó la Posición de Relación céntrica. La técnica para llegar a esta posición reproducible fue la de Jig de Lucia. Dicho desprogramador anterior fue elaborado por los estudiantes bajo la supervisión del docente a cargo de la cátedra. El desprogramador anterior fue utilizado por 15 minutos antes de la toma del registro interoclusal realizado con cera rosa doble templada con agua tibia. Los modelos montados fueron evaluados correctamente y los datos anotados en las fichas. Los datos fueron obtenidos a través de la observación de los modelos por separados, en Relación Céntrica, en posición intercuspal y en movimientos excéntricos para luego ser corroborados con el docente a cargo de la cátedra.

Como criterio de inclusión se presentaron como requisitos las siguientes características, estudiantes totalmente dentados en maxila y mandíbula, o con pérdida de pocas piezas dentarias que no afectaran al estudio de las variables observadas. Ausencia de síntomas de trastornos Temporomandibulares como dolor o crepitación. Ausencia de alte- raciones periodontales crónicas. En cuanto a antecedentes de tratamiento de ortodoncia fueron incluidos en la muestra todos los estudiantes que hayan empleado un tratamiento previo o no, con presencia o ausencia de contención fija o removible.

Como criterio de exclusión se menciona a estudiantes siguiendo tratamiento ortodóntico en el momento de la impresión dental, con enfermedades sistémicas, enfermedades de los tejidos orales o alergia a algún material de impresión o registro, los que cumplía con alguna de estas condiciones no fueron incluidos en la muestra. Se registraron los siguientes parámetros:

1. Forma del arco dental: Andrews determina que la quinta llave de la oclusión es la forma de los arcos dentales [9]. Estos arcos dentales obtienen su configuración según la forma del hueso de soporte, la erupción dental, músculos oro-faciales y fuerzas funcionales intraorales [10] Muchas clasificaciones se han desarrollado para describir el arco dental, pero generalmente se clasifican como triangular o forma de V, oval o circular y cuadrado [11].

2. Overbite o Sobre mordida vertical: La superposición vertical de los incisivos medidos en milímetro verticalmente desde el borde incisal del incisivo maxilar central derecho al borde incisal del incisivo mandibular derecho correspondiente. Los valores fueron separados en overbite normal: $1-3 \mathrm{~mm}$; mordida abierta $<0 \mathrm{~mm}$, mordida profunda $>4 \mathrm{~mm}$ [12].

3. Overjet o Sobremordida horizontal: se midió con regla milimetrada la distancia desde el punto más vestibular del borde incisal de los incisivos a la superficie más labial del incisivo mandibular correspondiente. El overjet se reportó como normal o ideal, cuando la relación de los incisivos superior e inferior en sentido horizontal no excedía los $3 \mathrm{~mm}$; aumentado cuando la distancia entre ambos incisivos excedía los $4 \mathrm{~mm}$; borde a borde si había contacto de los bordes incisales [13].

4. Coincidencia de la línea Media dentaria: fue determinada por una línea vertical ubicada entre los márgenes interproximales de los Incisivos Centrales Maxilares y 
se relacionó con la línea mediana interproximal de los incisivos centrales mandibulares cuando los modelos mantuvieran la posición de Máxima intercuspidación Habitual (MIH) [14].

5. Mordida cruzada: en la normalidad los dientes superiores se posicionan hacia el lado vestibular con relación a los inferiores. Registramos como mordida cruzada cuando los dientes mandibulares ocluyen por vestibular de los maxilares, según el lugar donde se ubica en la arcada puede ser anterior o posterior [15].

6. Clasificación de Angle: La Relación molar fue anotada para el lado izquierdo y derecho basado en el sistema de clasificación de Angle. La relación molar se registró como clase I cuando la cúspide mesiobucal del primer molar permanente maxilar ocluye con el surco mesiobucal del primer molar permanente mandibular a ambos lados. Una relación molar clase II o clase III se registró cuando la cúspide mesiobucal del primer molar permanente maxilar ocluye al menos medio ancho de la cúspide anterior o posterior al surco mesiobucal del primer molar permanente mandibular a ambos lados, respectivamente. Relación molar asimétrica se registró cuando los lados derechos e izquierdos muestran relación molar diferente. La Relación canina fue anotada para lado derecho e izquierdo. La Clase I canina cuando la punta del canino superior ocluye en la tronera entre el diente premolar canino y primer premolar mandibular a ambos lados. Una relación canina clase II o clase III se registró cuando la punta del canino superior ocluye anterior o posterior a la tronera entre el premolar canino y primer de la mandíbula en ambos lados, respectivamente. Relación canina asimétrica se registró cuando los lados derechos e izquierdos muestran diferente relación canina [9].

7. Los patrones de contacto dentario durante los movimientos laterales fueron anotados por un examinador entrenado. Los contactos del diente del lado de trabajo y de no trabajo durante la excursión mandibular lateral fueron verificados.

\section{Procesamiento de datos}

Los datos obtenidos fueron procesados y analizados con el programa estadístico Microsoft Excel 2016. Se realizaron tablas y gráficos de valores para las variables de forma del arco dental, mediciones de overbite y overjet, discrepancia de la línea media, mordida cruzada, clasificación de Angle molar y canina, patrón de desoclusión e interferencias del lado de no trabajo.

\section{Resultados y Discusión}

\section{Forma del Arco Dental}

La forma del arco dental más prevalente entre los alumnos del segundo año estudiados fue ovoide tanto para la arcada superior en un $83 \%$ como la inferior en un $73 \%$. La forma de arco cuadrada en el arco superior se presentó en un $12,2 \%$ y en el arco inferior en un $20,5 \%$. La forma de arco triangular fue escasamente registrada con un mismo porcentaje de 4,8\% en ambos arcos.

Estudios comparativos en México han reportado formas de arco cuadradas en indígenas y ovaladas en mestizos. Respecto a la población colombiana, resultados similares a los encontrados en este estudio fueron registrados, siendo los arcos ovalados los más prevalentes en las tres poblaciones estudiadas en indígenas del Amazonas Colombiano [10].

En un esudio en Corea, se seleccionaron 50 individuos de 24 a 32 años que visitaron la Universidad Dental de Kyung Hee Hospital en Gangdong, Seúl. La distribución de la forma de arco dental más prevalente fue también la forma ovoide (maxilar 52\% y 56\% de mandíbula), seguido por la forma de $\mathrm{V}$ o triangular y la forma de $\mathrm{U}$ o cuadrangular [16].
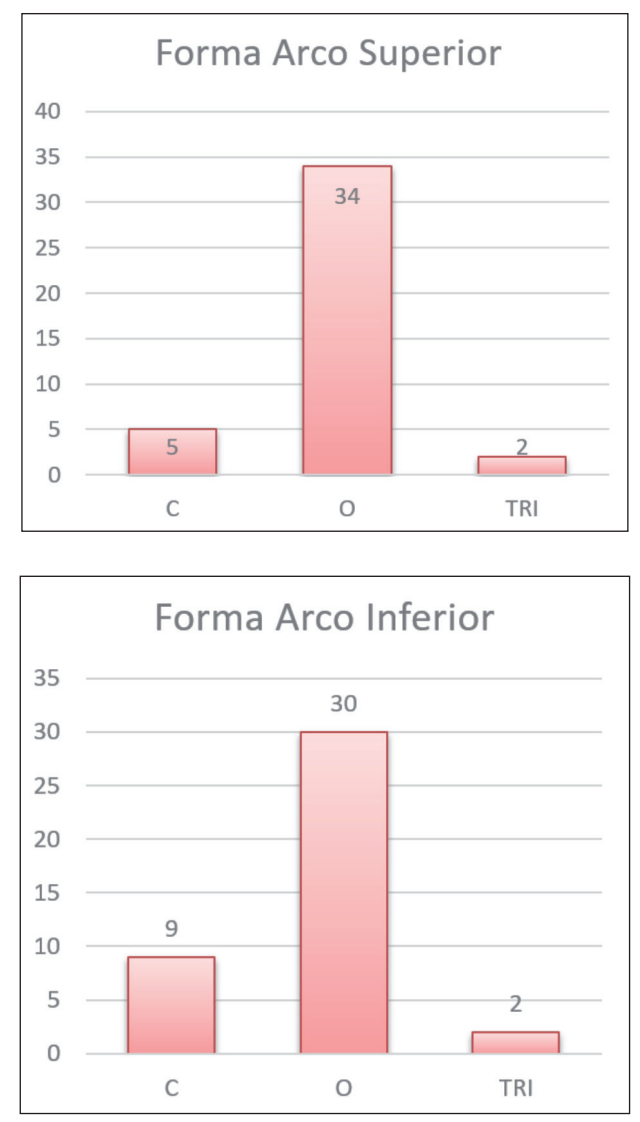

Figura 4: Gráficos de prevalencias de formas de arcos dentales superiores e inferiores.

\section{Overjet y Overbite}

El overjet resultó normal o ideal en 30 alumnos representando un $77,2 \%$ aumentado en $11(26,8 \%)$, y no se registró ningún caso de mordida borde a borde u overjet negativo. El overbite fue normal en 35 estudiantes $(85,4 \%)$, se presentó un overbite aumentado o mordida profunda en 6 de ellos $(14,6 \%)$ 
Resultados similares fueron encontrados en un estudio cuyo objetivo fue determinar la prevalencia de la maloclusión en adolescentes de Anatólia Central en donde fueron estudiadas 13 escuelas estatales de la cuidad de Kirikkale, localizada al sur de la capital de Turquía, siendo el overbite normal la característica más común $(73,5 \%)$; el overbite aumentado fue registrado en un $18,3 \%$ de la muestra. EL overjet normal estaba presente en un $64,5 \%$. La prevalencia de overjet aumentado fue de un $25,1 \%$; resultado mayor que la prevalencia del overjet negativo $(10,4 \%)$ [17].
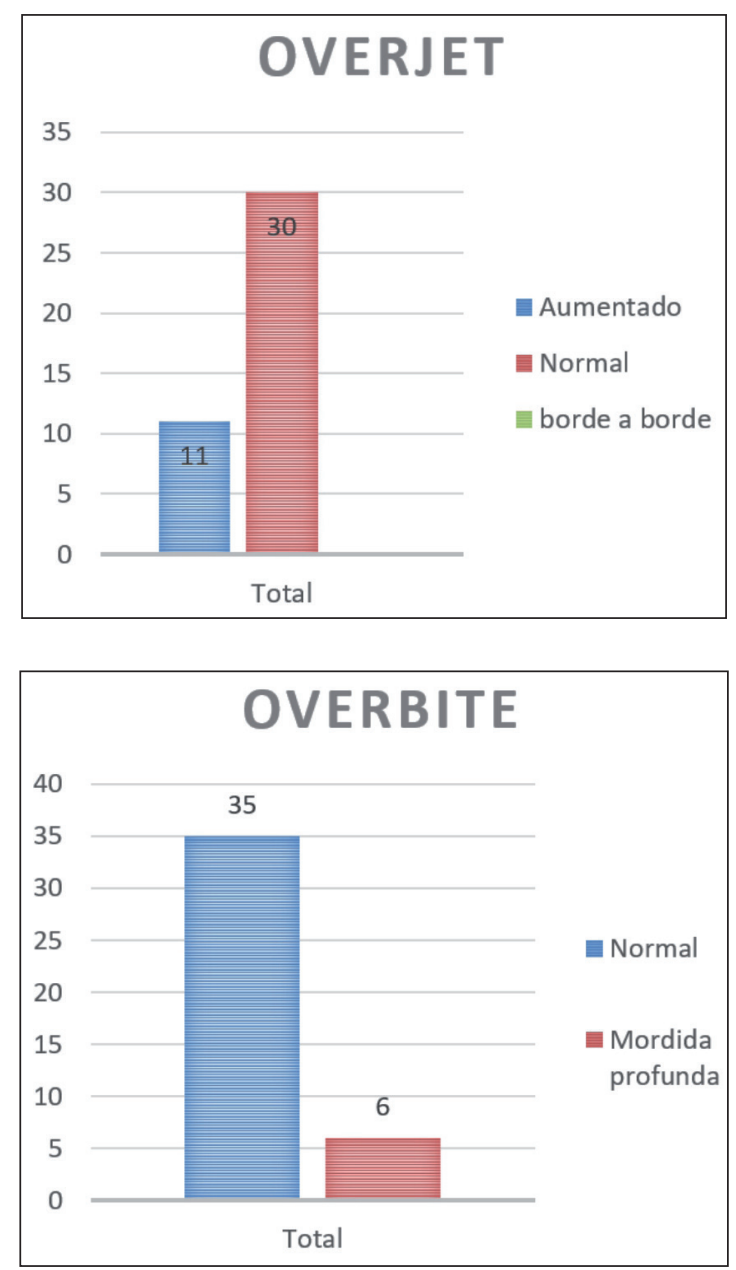

Figura 5: Resultados de Overje y Overbite.

\section{Coincidencia de la línea media dentaria}

Calificada como uno de los parámetros estéticos principales, la línea media dentaria, nos transmite una percepción de armonía (14). La discrepancia de la línea media se encontró en 22 de 41 sujetos (53,6\%). En un estudio similar sobre un total de 50 pacientes femeninas de 18 a 50 años en La Universidad Odontológica de Saveetha, se encontró resultados mayores de coincidencia de las líneas medias, $69 \%$ de las pacientes presentaban coincidencias de las líneas medias [18].

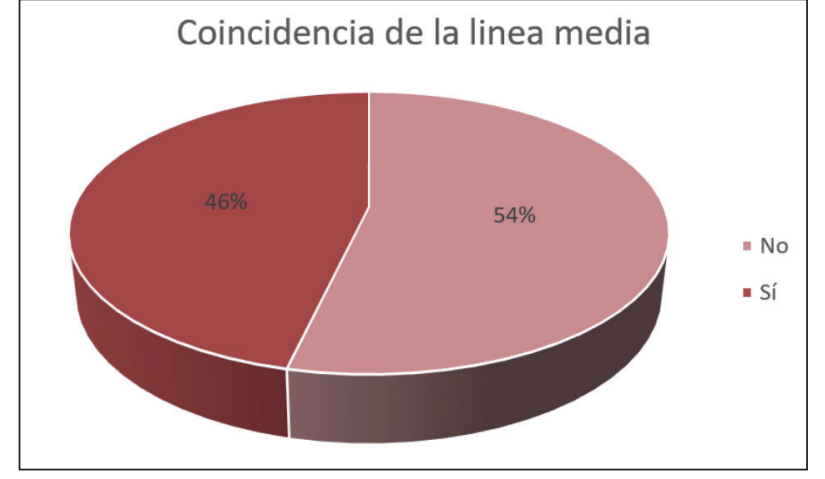

Figura 6: Coincidencia de la línea media maxilar y mandibular.

\section{Mordida Cruzada}

En este estudio, los casos de mordida cruzada son infrecuentes, se registró 4 estudiantes con mordida cruzada que corresponden al 9,7\%; de los cuales 3 presentaron mordida cruzada posterior izquierda $7,3 \%$ y 1 estudiante presentó mordida cruzada bilateral $2,4 \%$.

Datos aproximados fueron encontrados en un estudio que determinó la prevalencia de la maloclusión de adolescentes con edades entre 12,5 y 16,2 realizado entre mayo del 2008 y diciembte de 2012 en Anatólia Central, en donde fueron estudiadas trece escuelas estatales de la cuidad de Kirikkale, localizada al sur de la capital de Turquía donde la prevalencia de los valores de mordida cruzada fue de $8,2 \%$ de la muestra [17].

En un estudio realizado en cinco poblaciones indígenas de Amazonas semiaisladas $(n=351)$ fueron evaluados y comparados con datos publicados previamente de personas de Amazonas urbanas. Se encontró también una baja prevalencia de la mordida cruzada posterior en las aldeas de Koatinemo (4,6\%), Pat-Krô (3,5\%) y Ploughing-Laranjal $(2,3 \%)[5]$.

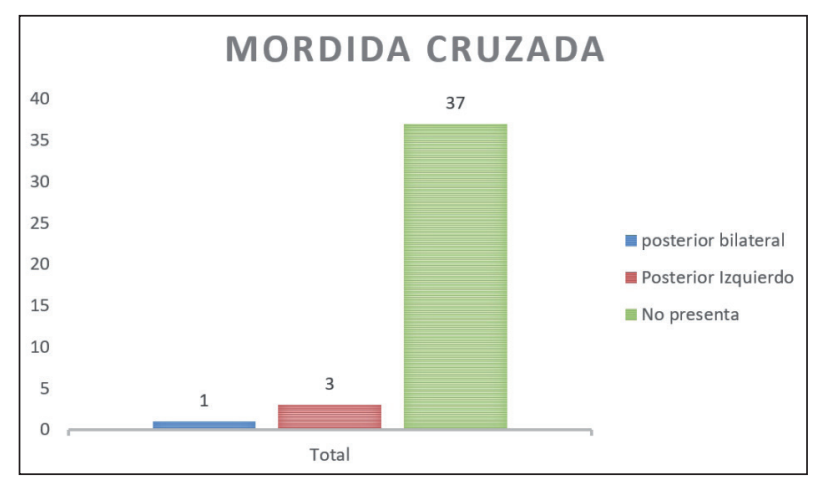

Figura 7: Resultado de Mordida Cruzada.

\section{Clasificación de Angle}

Los estudiantes presentaron en un 39\% Clase I Bilateral molar; de los cuales en la misma cantidad de estudiantes se registró la ausencia de por lo menos un molar 39\%, imposibilitando el registro de los mismos dentro de una 
clasificación bilateral de Angle; la Clase 2 Bilateral tuvo una prevalencia de 7,3\% y la Clase 3 Bilateral un 4,9\%; clases molares asimétricas fueron encontradas en un 9,8\%. En cuanto a la Clasificación Canina, se registró una prevalencia de $63,4 \%$ de Clase I bilateral, $19,5 \%$ de Clase II bilateral y $17,1 \%$ de asimetrías entre lados derechos e izquierdos.

En el Departamento de Medicina y Radiología Oral del Instituto Dasmesh de investigación y Ciencias Dentales, Faridkot, Punjab, India, doscientos cincuenta sujetos que acudieron al mismo en búsqueda de tratamiento de ortodoncia de junio de 2014 a enero de 2015 fueron seleccionados al azar e incluido en un estudio. En primer lugar, el tipo de maloclusión se examinó clínicamente en todos los sujetos y se clasificó de acuerdo con la clasificación de maloclusión de Angle. Los resultados encontrados difieren a los nuestros registrando para la clase II de Angle un $51,20 \%$ de los casos resultando la más común entre ambos sexos, seguida de la clase I en un $43,20 \%$ de los casos, mientras que la clase III solamente en un $5,60 \%$ de los casos siendo al igual que en nuestro estudio el tipo menos prominente [20].

Modelos de estudio de pretratamiento de 112 personas entre ellas 84 mujeres con edad media 19.77 años $\pm 4,86$ y 28 varones con edad media $20.07 \pm 4,45$ años fueron evaluados en un estudio que relacionó las características oclusales y el tamaño dentario en Malasia. La distribución de grupos étnicos fueron 50 chinos, 32 indios y 30 malayos y se agruparon de acuerdo a su maloclusión en $46.4 \%$ clase I, $33 \%$ clase II y $20,5 \%$ clase III, resultando también mayores los porcentajes de pacientes con Clase I [19].

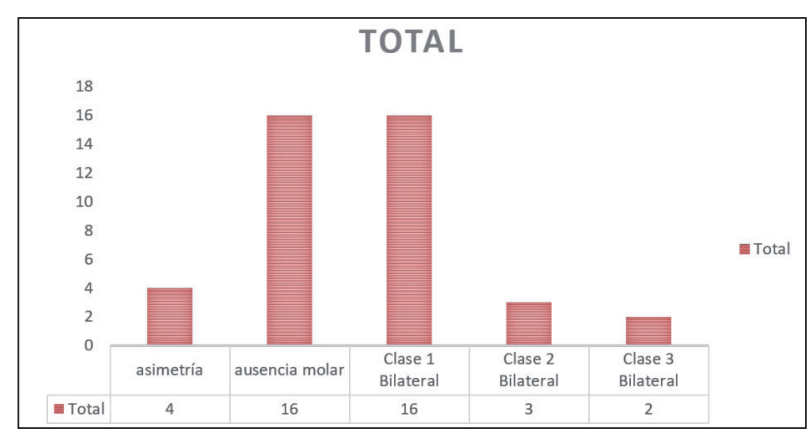

Figura 8: Clasificación Molar de Angle.

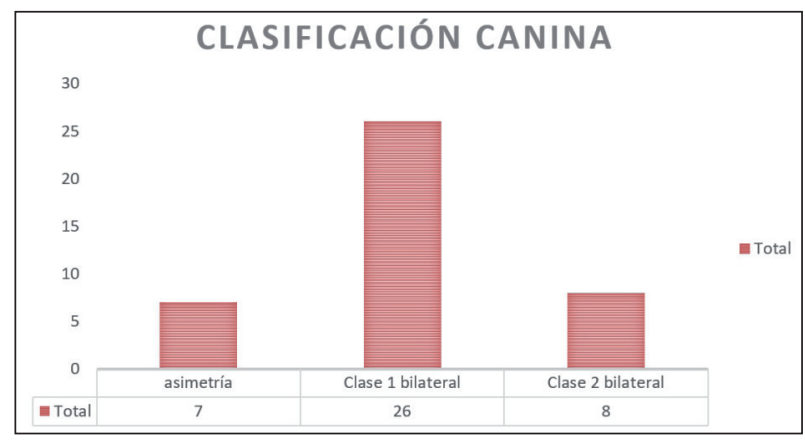

Figura 9: Clasificación Canina.

\section{Patrones de contacto dentario durante los movimientos laterales}

Entre los estudiantes se evidenciaron los siguientes esquemas oclusales para los movimientos laterales derechos e izquierdos respectivamente: oclusión protegida canina en el 58,5\% y el 56\%; La función de grupo fue encontrada en el $22 \%$ y el $8,9 \%$, interferencias en el $12,5 \%$ y el $24,4 \%$, los patrones de función de grupo anterior el 7,3\% y el 8,9\%.

Resultados diferentes fueron encontrados en un estudio presentado en el año 2015 por Francová, Eber y Zapletalová donde se analizaron los datos de 100 adultos jóvenes. En cuanto al contacto oclusal en el lado de trabajo la guía canina estaba presente en solamente en el $25 \%$ de las laterotrusiones; la función de grupo posterior fue la más prevalente y estuvo presente en el 56\% de laterotrusiones, la función de grupo anterior estaba presente en el 18,5\% de las laterotrusiones; Otros esquemas estaban presentes en el $0,5 \%$ de las laterotrusiones. Las interferencias estuvieron presentes en un porcentaje más bajo de $1 \%$ [20].

Similares resultados a los de Francová y colaboradores fueron encontrados un estudio que se realizó en el Departamento de Prostodoncia de la escuela de ciencias médicas de la Universidad de Katmandú con 80 sujetos sanos entre las edades de 18-30 años. Fueron seleccionados al azar de estudiantes de pregrado y de higienista dental del Colegio de ciencia dental con el consentimiento por escrito. Esta edad fue seleccionada debido al desgaste oclusal mínimo. Los patrones de la mayoría eran función de grupo (84\%), los patrones de guías caninas se encontraron que el 12\% y el patrón inclasificable se encontró que 4\% [21].

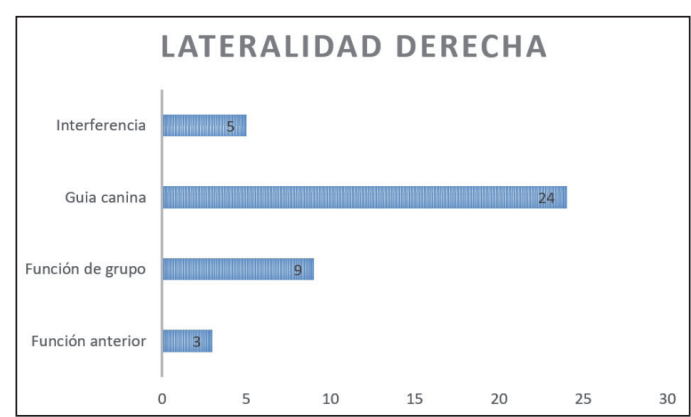

Figura 10: Patrones de desoclusión en estudiantes para lado de trabajo derecho.

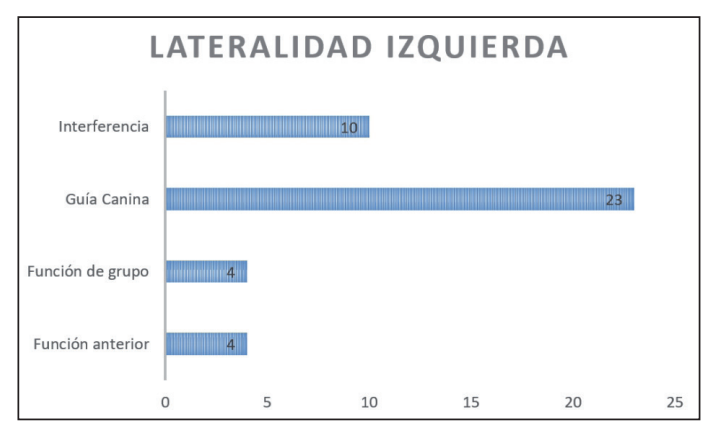

Figura 11: Patrones de desoclusión de estudiantes para lado de trabajo izquierdo. 


\section{Conclusiones}

Podemos destacar que, la forma del arco dental más prevalente entre los alumnos del segundo año estudiados fue la ovoide tanto para la arcada superior en un $83 \%$ como la inferior en un $73 \%$. Los resultados de las mediciones de overjet y overbite tuvieron una tendencia mayoritariamente hacia los parámetros normales. El overjet resultó normal o ideal en 30 alumnos representando un 77,2\%. El overbite fue normal en 35 estudiantes $(85,4 \%)$.

La discrepancia de la línea media está presente en aproximadamente la mitad de los alumnos, 22 de 41 sujetos $(53,6 \%)$.

Los casos de mordida cruzada son infrecuentes, registrándose solo en 4 estudiantes con mordida cruzada que corresponden al 9,7\%; de los cuales 3 presentaron mordida cruzada posterior izquierda $7,3 \%$ y 1 mordida cruzada bilateral $2,4 \%$.

La clase I de Angle molar y canina bilateral es la más frecuente. Los estudiantes presentaron en un 39\% Clase I Bilateral molar. En cuanto a la Clasificación Canina, se registró una prevalencia de $63,4 \%$ de Clase I bilateral.

La desoclusión canina fue el patrón más común de movimiento lateral funcional existiendo en un bajo porcentaje interferencias.

\section{Agradecimientos}

A la Carrera de Odontología de la Facultad de Ciencias de la Salud de la Universidad Católica "Nuestra Señora de la Asunción" Campus Itapúa, por el aporte de la infraestructura y los recursos humanos necesarios para el desarrollo de esta investigación.

\section{Referencias}

1. Prashant, M; Nilesh, M; Sumeet, M; Richa, M; Jyoti, R; Olavo, N. Prevalence of different types of malocclusion in young adults, in Ahmednagar District, Maharashtra (according to Angle's classification). Pravara Medical Review. 2018; 10(1): p. 4-9.

2. Sánchez-Tito, MA; Yañez-Chávez, EE. Association between facial biotipe and overbite: Pilot study. Rev. Estomatol. Herediana. 2015 January; 25(1).

3. Meneses-Gómez, E; Vivares-Builes, A; Janeth-Rodríguez, M. Perfil epidemiológico de la oclusión estática y hábitos orales en un grupo de escolares de la ciudad de Medellín. Revista Nacional de Odontología. January 2016; 12(22).

4. Normando, D; Almeida, MAO; Quintão, CCA. Dental crowding The role of genetics and tooth wear. Angle Orthodontist. 2013 Enero; 83(1): p. 10-15.

5. De Souza, B; Bichara, L; Guerreiro, J; Quintão, C; Normando, D. Occlusal and facial features in Amazon indigenous: An insight into the role of genetics and environment in the etiology dental malocclusion. Archives of Oral
Biology. 2015 Sep; 60(11): p. 77-86.

6. Manfredini, D; Vano, M; Peretta, R; Guarda-Nardini, L. Jaw clenching effects in relation to two extreme occlusal features: patterns of diagnoses in a TMD patient population. CRANIO: The Journal of Craniomandibular \& Sleep Practice. 2014 January 1; 32(1): p. 45.

7. Manfredini, D SemrrgnI[[2M1, Av. Are occlusal features associated with different temporomandibular disorder diagnoses in bruxers? Cranio-The Journal of Craniomandibular Practice. 32(4): p. 283-8.

8. Manfredini, D; Perinetti, G; Guarda-Nardini, L. Dental malocclusion is not related to temporomandibular joint clicking: a logistic regression analysis in a patient population. Angle Orthodontist. 2014 Marzo; 84(2): p. 310.

9. Manns, A; Jorge, B. Manual Práctico de Oclusión Dentaria. Segunda ed. Rafael AJ, editor. Caracas: Amolca; 2006.

10. Asiry, M. Occlusal Status among 12-16 Year-Old School Children in Riyadh, Saudi Arabia. Journal of International Oral Health. 2015 May; 7(5): p. 20.

11. Bedoya-Rodríguez, A; Montoya-Gómez, J; González-Benavidez, V; Tamayo-Cardona, J; Martínez-Cajas, C. Forma y tamaño del arco dental en poblaciones de tres ascendencias étnicas colombianas. CES Odontología. 2016 July; 29(2): p. 1-12.

12. Paranhos, LR; Adilson, LR; de Novaes Benedicto, E; Àvila Maltagliati, L; de Almeida Cardoso, M; Capelozza Filho, L. Is there any association between facial type and mandibular dental arch form in subjects with normal occlusion? Acta Scientiarum: Health Sciences. 2014; 36(1): p. 129-134.

13. Farheen, F; Mubassar, F; Attiya, $\mathbf{s}$. Reliability of overbite depth indicator (ODI) and anteroposterior dysplasia indicator (APDI) in the assessment of different vertical and sagittal dental malocclusions: a receiver operating characteristic (ROC). Dental Press J. Orthod. 2016 December; 21(5): p. 75-81.

14. Giraldo-Mejía, A; Parra-Ramírez. Características dentales y óseas en un grupo de escolares del área urbana de Manizales según género y tipo de dentición. GCES Odontología. January 2014; 27(1): p. 30-43.

15. Weber, B; Fuentes, R; García, N; Cantín, M. Relaciones de Forma y Proporción del Incisivo Central Maxilar con Medidas Faciales, Linea Mediana Dentaria y Facial en Adultos. International Journal of Morphology. 2014 September; 32(3): p. 1101-4.

16. Segura Martínez, N; Medrano Montero, J; Moreira Segura, F; Segura Martínez, N; Terán Pérez, s. Prevalencia de mordida cruzada en pacientes de la Clínica Estomatológica Artemio Mastrapa / Prevalence of Crossbite in Patients of Artemio Mastrapa Dental Clinic. Correo Científico Médico. 2017 June; 21(2).

17. Park, SJ; Leesungbok, R; Song, JW; Chang, S; Lee, sW; Ahn, SJ. Analysis of dimensions and shapes of maxillary and mandibular dental arch in Korean young adults. The Journal Of Advanced Prosthodontics. 2017 October; 9(5): p. 321-7. 
18. Bílgíç, F; Gelgor, I; Celebi, A. Prevalência de má oclusão e necessidade de tratamento ortodôntico em adolescentes da Anatólia Central comparados a adolescentes europeus de outras nacionalidades. Dental Press Journal of Orthodontics. 2015 November; 20 (6): p. 75-81.

19. Priyadharshni, S; Felicita, A. Prevalence of maxillary midline shift in female patients reported to Saveetha Dental College. Drug Invention Today. 2019 January; 11(1): p. 77-80.

20. Mulimani, P; Azmi, M; Jamali, N; Basir, N; Soe, H. Bolton's tooth size discrepancy in malaysian orthodontic patients: Are occlusal characteristics such as overjet, overbite, midline, and crowding related to tooth size discrepancy in specific malocclusions and ethnicities? APOS Trends in Orthodontics. 2018 October-December; 8(4).
21. Sapkota, B; Gupta, A. Pattern of Occlusal Contacts in Lateral Excursions (Canine Protection or Group Function). KUMJ. 2015 October 12; 12(1): p. 43-7.

22. Francová, K; Eber, M; Zapletalová, J. Clinical Study: Functional occlusal patterns during lateral excursions in young adults. The Journal of Prosthetic Dentistry. 2015 June 1; 113(6): p. 571-7.

23. M.K. krokida; Kouris, DM. Rehydratation Kinetics of dehydrated products. Journal of food Engineering. 2003. 